\title{
Conformal Dimensions via Large Charge Expansion
}

\author{
Debasish Banerjee, ${ }^{1, *}$ Shailesh Chandrasekharan, ${ }^{2, \dagger}$ and Domenico Orlando ${ }^{3,4, \$}$ \\ ${ }^{1}$ NIC, DESY, Platanenallee 6, D-15738 Zeuthen, Germany \\ ${ }^{2}$ Department of Physics, Duke University, Durham, North Carolina 27708, USA \\ ${ }^{3}$ Albert Einstein Center for Fundamental Physics, Institute for Theoretical Physics, \\ University of Bern, Sidlerstrasse 5, CH-3012 Bern, Switzerland \\ ${ }^{4}$ INFN, Sezione di Torino, Via Pietro Giuria 1, 10125 Torino, Italy
}

(Received 22 August 2017; revised manuscript received 19 December 2017; published 9 February 2018)

\begin{abstract}
We construct an efficient Monte Carlo algorithm that overcomes the severe signal-to-noise ratio problems and helps us to accurately compute the conformal dimensions of large- $Q$ fields at the Wilson-Fisher fixed point in the $O(2)$ universality class. Using it, we verify a recent proposal that conformal dimensions of strongly coupled conformal field theories with a global $U(1)$ charge can be obtained via a series expansion in the inverse charge $1 / Q$. We find that the conformal dimensions of the lowest operator with a fixed charge $Q$ are almost entirely determined by the first few terms in the series.
\end{abstract}

DOI: 10.1103/PhysRevLett.120.061603

Conformal field theories (CFTs) occupy a central place in our understanding of modern physics. They describe critical phenomena in condensed matter physics and statistical models [1,2], quantum gravity via the AdS/ CFT correspondence [3], and can be found at fixed points of renormalization group flows [4-7]. They are uniquely described by a set of dimensionless numbers (the CFT data), i.e., conformal dimensions and operator product expansion (OPE) coefficients associated with the primary fields of the theory. Since they are typically strongly coupled and lack a characteristic scale, it is often difficult to compute the conformal dimensions analytically. Still, a number of sophisticated techniques have been developed to deal with this challenge, both perturbatively (e.g., 4- $\epsilon$ expansion, fixed-dimension expansion, and large- $N$ limit; see [2] for a review) and nonperturbatively (e.g., bootstrap [8]). In some cases Monte Carlo (MC) techniques offer a reliable numerical approach for computing the conformal dimensions $[9,10]$.

Energies of low-lying states also capture universal features of a $2+1$ dimensional CFT when the theory is studied on a space-time manifold $\mathbb{R} \times \Sigma$ (see [11,12] for some recent work in this direction). For example, conformal dimensions $D$ of operators on $\mathbb{R}^{3}$ are related to the energies $E_{S^{2}}$ of states living on a two-sphere of radius $r_{0}$ through the relation $D=r_{0} E_{S^{2}}[13,14]$. This relation, known as the state-operator correspondence, is a

Published by the American Physical Society under the terms of the Creative Commons Attribution 4.0 International license. Further distribution of this work must maintain attribution to the author(s) and the published article's title, journal citation, and DOI. Funded by SCOAP . consequence of the fact that $\mathbb{R}^{3}$ is conformally equivalent to $\mathbb{R} \times S^{2}\left(r_{0}\right)$. Recently, such a connection has been used in CFTs with global $U(1)$ charges to show that the conformal dimension $D(Q)$ of the lowest operator with fixed $U(1)$ charge $Q$ can be expanded in inverse powers of the charge density on a unit sphere $Q / 4 \pi[15,16]$ (see also [17-21] for related work),

$D(Q)=\sqrt{\frac{Q^{3}}{4 \pi}}\left[c_{3 / 2}+c_{1 / 2}\left(\frac{4 \pi}{Q}\right)+\cdots\right]+c_{0}+\mathcal{O}\left(\frac{1}{Q}\right)$,

where $c_{0} \approx-0.094$ [22] and the other coefficients only depend on the universality class. While a simple dimensional analysis allows one to predict the leading large- $Q$ behavior, it is a priori unclear if a power series can capture the subleading corrections. Recent work argues that by separating the theory into sectors of fixed charge $Q$ one can construct an effective field theory (EFT) in each sector, which can be used to compute the energies as a power series in $1 / Q$. Through the state-operator correspondence one can then obtain the series expansion of the conformal dimension $D(Q)$ and directly relate the coefficients in Eq. (1) to the coefficients in the energy expansion.

In this Letter we make significant progress in establishing that Eq. (1) is an excellent description of the WilsonFisher (WF) fixed point in the $O(2)$ universality class. In order to achieve this we overcome severe signal-to-noise ratio problems in Monte Carlo methods that usually hinder calculations of $D(Q)$ for large values of $Q$. Our new approach allows us to determine the corresponding universal coefficients $c_{3 / 2}=1.195(10), c_{1 / 2}=0.075(10)$ for the first time. In Fig. 1 we demonstrate that the measured values of $D(Q)$ using our Monte Carlo approach are 


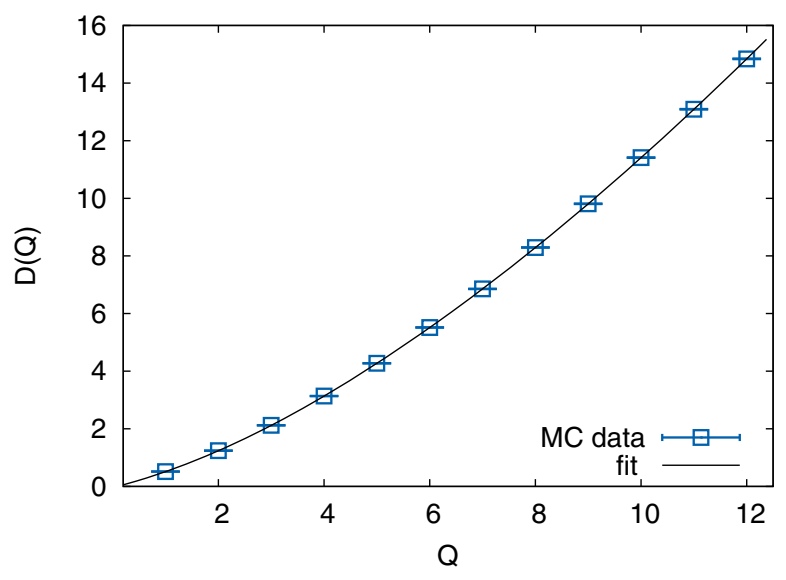

FIG. 1. Plot of the values of $D(Q)$ extracted from our Monte Carlo calculations at the $O(2) \mathrm{WF}$ fixed point, along with the plot of Eq. (1) (solid line) with our estimated values $c_{3 / 2}=1.195$ and $c_{1 / 2}=0.075$ and previously computed value $c_{0}=-0.094$. It is surprising that these three leading coefficients in Eq. (1) can predict the conformal dimensions for all $Q \geq 1$ very well.

excellently described by Eq. (1). Surprisingly, we find that the large- $Q$ expansion with the first three coefficients matches the conformal dimensions even at $Q=1$, within a few percent. Thus, our work demonstrates that at least in some class of models, the large- $Q$ expansion is similar to the $\epsilon$ expansion in the fact that the strongly coupled conformal fixed point can be described within a simple perturbative framework. The coefficients of the expansion could still be difficult to compute analytically, but perhaps bootstrap techniques could be developed for it [23].

To understand the origin of Eq. (1), consider the conformal field theory describing the Wilson-Fisher fixed point in the three-dimensional $O(2)$ universality class. In a fixed-charge sector $Q$, the charge density introduces the mass scale $\sqrt{Q / V}$ in the theory and, hence, for momentum scales $p \ll \sqrt{Q / V}$ the physics is described by a Goldstone field $\chi$ that is controlled by an approximately scaleinvariant Lagrangian [15,17] (see also [24] for a related approach to effective descriptions of nonrelativistic CFTs),

$$
L=\frac{k_{3 / 2}}{27}\left(\partial_{\mu} \chi \partial^{\mu} \chi\right)^{3 / 2}+\frac{k_{1 / 2} R}{3}\left(\partial_{\mu} \chi \partial^{\mu} \chi\right)^{1 / 2}+\cdots
$$

where $R$ is the scalar curvature of the manifold $\mathbb{R} \times \Sigma$. Thus, we learn that in the large- $Q$ limit only the two parameters $k_{3 / 2}$ and $k_{1 / 2}$ that appear in Eq. (2) play an important role; all other terms are suppressed [16,18]. Since the charge is nonzero, the action is only meaningful away from $\chi=0$ and is to be expanded around the fixed-charge homogeneous classical solution $\chi=\mu t$. Using the effective quantum Hamiltonian arising from the effective Lagrangian Eq. (2), one can show that the total energy of the system is given by
$E_{\Sigma}(Q)=\sqrt{\frac{Q^{3}}{V}}\left[c_{3 / 2}+c_{1 / 2}\left(\frac{R V}{2 Q}\right)+\cdots\right]+q_{\Sigma}+\mathcal{O} \frac{1}{Q}$,

where the first two terms are related to the couplings in the effective Lagrangian Eq. (2) through the relations $k_{3 / 2}=$ $4 / c_{3 / 2}^{2}$ and $k_{1 / 2}=-c_{1 / 2} / c_{3 / 2}$. The higher-order terms in the expansion are related to higher-dimensional operators in Eq. (2) and quantum corrections. The last term $q_{\Sigma}$ arises due to quantum fluctuations that can be computed exactly for simple manifolds. For the sphere $\left(R=2 / r_{0}^{2}\right)$ one finds $q_{S^{2}}=c_{0} / r_{0}$ where $c_{0} \approx-0.094$ [22], while for the torus $(R=0)$ it is $q_{T^{2}}=c_{0} / L$ with $c_{0} \approx-0.508$ [25].

By choosing $\Sigma=S^{2}$ and using the state-operator correspondence one can now easily derive Eq. (1). It is interesting to note that the coefficients $c_{3 / 2}, c_{1 / 2}$ in Eqs. (1), (3) are related to the low-energy constants $k_{3 / 2}$ and $k_{1 / 2}$ of the effective Lagrangian in Eq. (2). Indeed, these low-energy constants are independent of the manifold chosen and depend only on the CFT. Assuming the manifold is the torus we predict that

$$
\lim _{Q \rightarrow \infty} \frac{D(Q)}{E_{T^{2}}(Q) L}=\frac{1}{2 \sqrt{\pi}} .
$$

Note that every term in the energy expansion is a dimensionless function of three variables: a coefficient in the $D(Q)$ expansion, a geometrical term from the manifold, and a power of $V / Q$.

The motivation of our current work is to compute $D(Q)$ and $E_{\Sigma}(Q)$ in the classical $O(2) \sigma$ model on a torus and verify the expansions in Eqs. (1) and (3) and the connections between them. We accomplish this by regularizing the classical $O(2) \sigma$ model on a cubic lattice with lattice spacing $a$ and use Monte Carlo methods to perform the calculations. The model is defined by phases, $\exp \left(i \theta_{x}\right)$, on each three-dimensional lattice site $x=\left(x_{1} a, x_{2} a, x_{3} a\right)$ and the nearest-neighbor action

$$
S=-\beta \sum_{x, \alpha} \cos \left(\theta_{x}-\theta_{x+\hat{\alpha} a}\right)
$$

Here $\hat{\alpha} a$ denotes the three unit lattice vectors, and $\beta$ is the coupling of the model. The physics of the Wilson-Fisher fixed point can be studied by tuning the coupling to its critical value $\left(\beta_{c}=0.4541652\right.$ [26-28]), where a secondorder phase transition separates the symmetric phase $\left(\beta<\beta_{c}\right)$ and the spontaneously broken superfluid phase $\left(\beta>\beta_{c}\right)$. Universality implies that details of our specific model should be irrelevant in the limit $a \rightarrow 0$ which is naturally reached by studying large lattices at $\beta_{c}$.

Configurations that contribute to the partition function of the lattice model at the critical point can be efficiently generated by both the Wolff cluster algorithm [29] and the 
TABLE I. Conformal dimensions $D(Q)$ obtained previously by other methods for $Q \leq 4$. Field theory results in $4-\epsilon$ dimensions at five loops are in the second column, six-loop results at $d=3$ are in the third column ([31] for $Q=2$, in [32] for $Q=3$ and in [33] for $Q=4$ ), previous MC results are in the fourth column [34], and bootstrap results are in the fifth column [35].

\begin{tabular}{llllc}
\hline \hline$Q$ & \multicolumn{1}{c}{$\epsilon^{5}$} & \multicolumn{1}{c}{$\lambda^{6}$} & \multicolumn{1}{c}{ MC } & Bootstrap \\
\hline 1 & $0.518(1)$ & $\ldots$ & $0.5190(1)$ & $0.5190(1)$ \\
2 & $1.234(3)$ & $1.23(2)$ & $1.236(1)$ & $1.236(3)$ \\
3 & $2.10(1)$ & $2.10(1)$ & $2.108(2)$ & $\ldots$ \\
4 & $3.114(4)$ & $3.103(8)$ & $3.108(6)$ & $\ldots$ \\
\hline \hline
\end{tabular}

worm algorithm based on the worldline representation [30]. However, in order to compute the conformal dimension $D(Q)$ in $\mathbb{R}^{3}$ we need to compute the two-point correlation function $C_{Q}(r)$ of charge $Q$ fields on a large lattice of size $L$, which is expected to decay as a power law for large separations $r \ll L$ at the critical point,

$$
C_{Q}(r)=\left\langle\exp \left(i Q \theta_{\mathrm{r}}\right) \exp \left(-i Q \theta_{0}\right)\right\rangle \sim \frac{a(Q)}{|\mathbf{r}|^{2 D(Q)}}
$$

Fitting the data to this form we can in principle extract $D(Q)$ and thus verify Eq. (1). Note that for $Q=1$, it reduces to the standard two-point correlation function, which is used to extract the critical exponent $\eta$ through the relation $C_{1}(r)=G(r) \propto 1 / r^{d-2+\eta}$. For $Q=2,3,4$, the corresponding conformal dimensions have also been computed earlier using different methods, and the results are summarized in Table I. Unfortunately, calculations of $D(Q)$ for higher values of $Q$ do not exist and, hence, the relation (1) remains unconfirmed.

It is difficult to measure $D(Q)$ for large values of $Q$ through a Monte Carlo method due to severe signal-tonoise ratio problems in the Monte Carlo calculations. With the Wolff cluster algorithm it is difficult to average numbers of order 1 to compute a small value of $C_{Q}(r)$ at large separations. In contrast, in the worm algorithm, it is difficult to correctly build the worldline configurations that contribute to the correlation function in the presence of charged sources $Q$ and $-Q$ separated by a large distance. In this case the severe signal-to-noise ratio problem emerges as an overlap problem between the vacuum ensemble and the one containing the sources. In order to overcome this problem we have designed an algorithm to efficiently compute the ratio

$$
R(L / 2)=\frac{C_{Q}(r=L / 2)}{C_{Q-1}(r=L / 2)}
$$

on cubic lattices of side $L$ for $8 \leq L / a \leq 120$ at the critical point $\beta_{c}$ (the details of our algorithm can be found in the Supplemental Material [36]). Using $R(L / 2)$ it is easy to

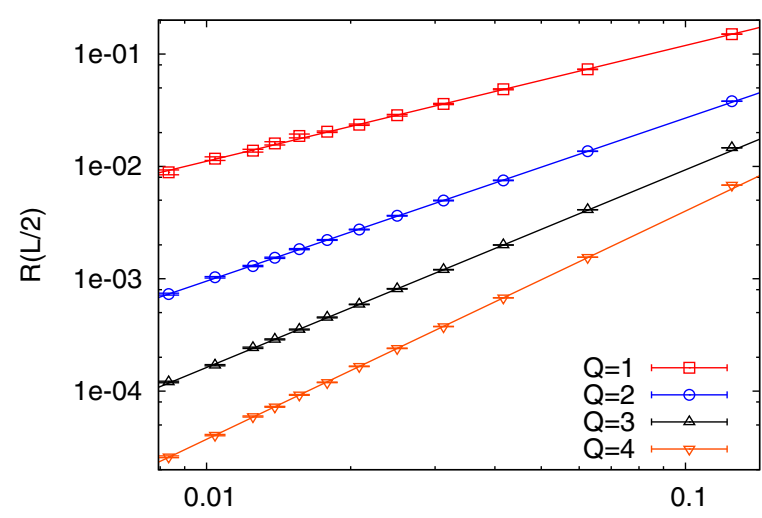

$1 / L$

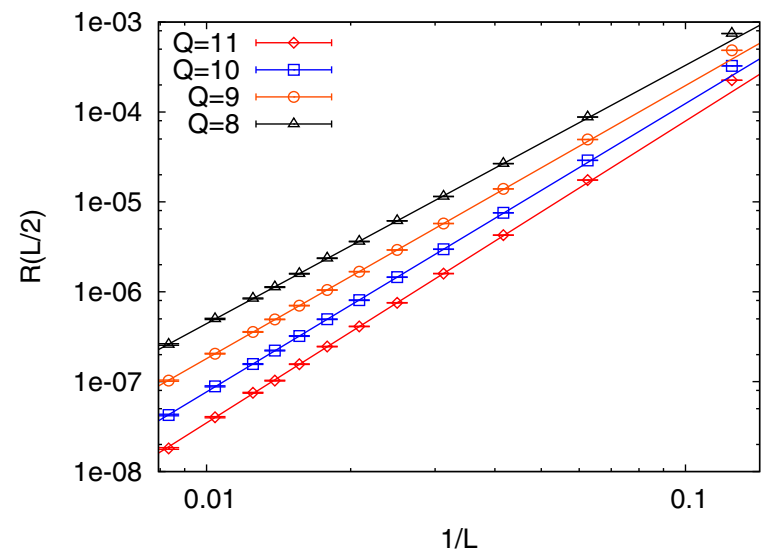

FIG. 2. The figures (top and bottom) show the quantity $R(L / 2)$ for different lattice sizes $L / a=8, \ldots, 120$ and different $Q$ values. The straight line on a log-log plot is indicative of the power law behavior, and the slope gives the difference of the conformal dimensions $2 \Delta(Q)$. Note that there is no visible signal-to-noise problem in these correlators.

extract the difference $\Delta(Q)=D(Q)-D(Q-1)$ using the relation $R(L) \sim 1 / L^{2 \Delta(Q)}$. The accuracy with which we are able to compute the ratio $R(L / 2)$ for various values of $Q$ can be seen in Fig. 2. Once the differences $\Delta(Q)$ are known, we can also extract $D(Q)$ by setting $D(Q=0)=0$. Our estimates of both $\Delta(Q)$ and $D(Q)$ using Monte Carlo calculations, are given in Table II. It is

TABLE II. Results for the conformal dimensions $\Delta(Q)$ and $D(Q)$ defined through (6). Fit systematics are discussed in the Supplemental Material [36]. While our results for $Q<4$ are in good agreement with previous results as seen in Table I, there is a slight deviation for $Q=4$.

\begin{tabular}{lccrcc}
\hline \hline$Q$ & $\Delta(Q)$ & $D(Q)$ & $Q$ & $\Delta(Q)$ & \multicolumn{1}{c}{$D(Q)$} \\
\hline 1 & $0.516(3)$ & $0.516(3)$ & 7 & $1.332(5)$ & $6.841(8)$ \\
2 & $0.722(4)$ & $1.238(5)$ & 8 & $1.437(4)$ & $8.278(9)$ \\
3 & $0.878(4)$ & $2.116(6)$ & 9 & $1.518(2)$ & $9.796(9)$ \\
4 & $1.012(2)$ & $3.128(6)$ & 10 & $1.603(2)$ & $11.399(10)$ \\
5 & $1.137(2)$ & $4.265(6)$ & 11 & $1.678(5)$ & $13.077(11)$ \\
6 & $1.243(3)$ & $5.509(7)$ & 12 & $1.748(5)$ & $14.825(12)$ \\
\hline \hline
\end{tabular}




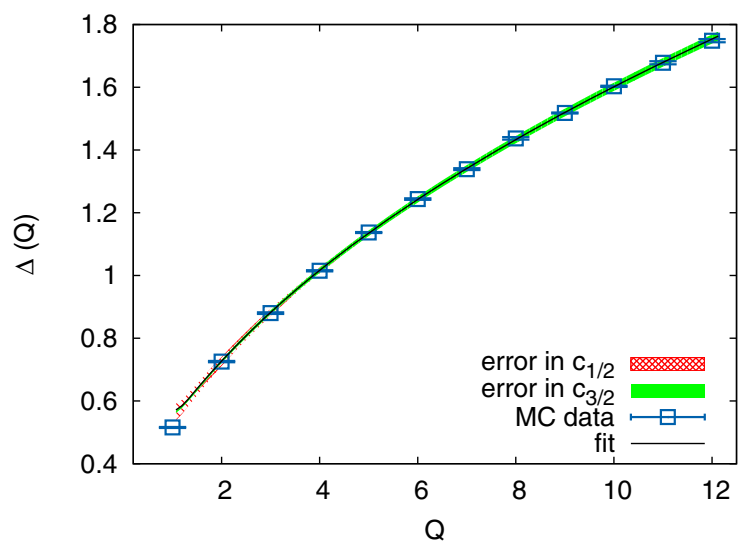

FIG. 3. Plot of $\Delta(Q)$ extracted using Monte Carlo calculations, along with a plot of Eq. (1) keeping the first three nonzero coefficients estimated using our fits.

easy to verify that our results match quite well with previous results in Table I when $Q<4$.

We can now verify if the conformal dimensions in Table II are consistent with Eq. (1). For this purpose we perform a combined fit of our data for the difference $\Delta(Q)$ and $D(Q)$ assuming that $c_{3 / 2}, c_{1 / 2}, c_{-1 / 2}$ are nonzero and $c_{0}=-0.094$ as expected. Taking into account various systematic errors from fitting procedures, we estimate $c_{3 / 2}=1.195(10)$, $c_{1 / 2}=0.075(10)$ and $c_{-1 / 2}=0.0002(5)$. The raw data for $\Delta(Q)$ are shown in Fig. 3, and further technical details are discussed in the Supplemental Material [36]. We also show a comparison with the prediction obtained by just keeping the first three leading terms of the expansion in Eq. (1). As the figure shows, this prediction works even at small values of $Q$ but is off only by a few percent at $Q=1$.

Next we explore if we can connect our above calculations of $c_{3 / 2}$ and $c_{1 / 2}$ with the ones appearing in Eq. (3) for the expansion of the energy on a torus. Lattice calculations naturally lead to a torus geometry in the continuum limit if we keep the physical length $L$ fixed while taking the number of lattice points in each direction, $L / a$, to infinity. The lattice spacing $a$ itself is defined by setting the lattice energy $E^{L}(Q)$ to be equal to the continuum energy $E_{T^{2}}(Q)$ on the torus of side $L$ as the continuum limit is taken. On the lattice we measure the energy in terms of the dimensionless number $E^{L}(Q) a_{t}$ as a function of $L / a$, where $a_{t}$ is the temporal lattice spacing. Although the lattice calculation at a fixed $L / a$ and charge $Q$ breaks the symmetry between space and time, in the continuum limit (i.e., $L / a \rightarrow \infty$ ), we expect $a_{t} / a \rightarrow 1$ due to the cubic symmetry of the lattice action, Eq. (5). Thus on the lattice with a fixed $L / a$ we can replace Eq. (3) by the formula

$E^{L}(Q) L=Q^{3 / 2}\left(c_{3 / 2}^{L}+c_{1 / 2}^{L} Q^{-1}+c_{-1 / 2}^{L} Q^{-2} \ldots\right)+q^{L}$,

such that in the continuum limit (i.e., large lattices) we expect this equation to turn into Eq. (3) on the torus, with
TABLE III. Values of $\Delta E^{L}(Q) L$ obtained on the lattice using the Monte Carlo method for various values of $Q$ on lattice sizes $L / a=8$ and 10 .

\begin{tabular}{llllll}
\hline \hline & \multicolumn{2}{c}{$\Delta E^{L}(Q) L$} & & \multicolumn{2}{c}{$\Delta E^{L}(Q) L$} \\
\cline { 2 - 3 } \cline { 5 - 6 }$Q$ & $L / a=8$ & $L / a=10$ & $Q$ & $L / a=8$ & $L / a=10$ \\
\hline 1 & $1.3442(5)$ & $1.3393(7)$ & 10 & $5.7135(24)$ & $5.6998(3)$ \\
2 & $2.2422(3)$ & $2.2311(4)$ & 11 & $6.0074(24)$ & $5.9960(4)$ \\
3 & $2.9012(4)$ & $2.8851(3)$ & 12 & $6.2866(24)$ & $6.2786(4)$ \\
4 & $3.4434(3)$ & $3.4259(3)$ & 13 & $6.5529(24)$ & $6.5487(4)$ \\
5 & $3.9142(3)$ & $3.8949(2)$ & 14 & $6.8078(32)$ & $6.8074(5)$ \\
6 & $4.3346(16)$ & $4.3150(2)$ & 15 & $7.0524(32)$ & $7.0560(20)$ \\
7 & $4.7178(16)$ & $4.6987(2)$ & 16 & $7.2884(32)$ & $7.2970(10)$ \\
8 & $5.0722(16)$ & $5.0545(3)$ & 17 & $7.5152(32)$ & $7.5280(20)$ \\
9 & $5.4026(16)$ & $5.3868(3)$ & 18 & $\cdots$ & $7.7530(20)$ \\
\hline \hline
\end{tabular}

$E^{L}(Q) \rightarrow E_{T^{2}}(Q), c_{3 / 2}^{L} \rightarrow c_{3 / 2}, c_{1 / 2}^{L} \rightarrow\left(R L^{2} / 2\right) c_{1 / 2}=0$, and $q^{L} \rightarrow q_{T^{2}}=-0.508$.

Unfortunately, computing $E^{L}(Q)$ on the lattice is subtle due to the additive renormalization of lattice energies [37-39]. Thus, in this Letter we use the techniques discussed in [30] to compute energy differences $\Delta E^{L}(Q)=$ $\left[E^{L}(Q)-E^{L}(Q-1)\right]$ at a fixed lattice size $L / a$. The idea is to couple a chemical potential $\mu a_{t}$ to the conserved charge $Q$ and extract the energy differences between ground states with charges $Q$ and $Q-1$ by tuning the chemical potential. At the critical chemical potential $\mu_{c}^{(Q-1)}=\Delta E^{L}(Q)$, the average charge of the system jumps from $Q-1$ to $Q$. Further details on the method can be found in the Supplemental Material [36].

Unfortunately, our method is efficient only on small lattice sizes $L / a$, limiting the range of $Q$ s that can be used. Remember that the EFT description is valid only when $1 \ll(L / a) / \sqrt{Q} \ll L / a$. Further, small lattices also imply larger lattice spacing errors, which means $c_{3 / 2}^{L}$ and $c_{1 / 2}^{L}$ may not quite agree with continuum expectations discussed above. Still, we can learn about the magnitude of the errors. With this in mind we have computed $\Delta E^{L}(Q) L$ in the range $1 \leq Q \leq 18$ for $L / a=8$ and 10 . Our results are tabulated in Table III.

Our data for $L / a=8$ and 10 fit well to Eq. (8) with a $\chi^{2} / \mathrm{DOF} \approx 1$, as long as we restrict the fits to the range $5 \leq Q \leq 13$ and $10 \leq Q \leq 18$, respectively. The fit result for $L / a=10$ is shown in Fig. 4 . The fits give $c_{3 / 2}^{L}=$ $1.235(10)$ and $c_{1 / 2}^{L}=0.12(10)$ for both sets of the data. However, $c_{-1 / 2}^{L}$ fits to $1.9(5)$ at $L / a=10$ and to $0.7(2)$ at $L / a=8$. Note that $c_{3 / 2}^{L}$ is only about $3 \%$ off from $1.195(10)$ extracted earlier using conformal dimensions. This error seems reasonable given our small lattices (see the Supplemental Material for further discussion of this point [36]). The coefficient $c_{1 / 2}^{L}$ is small, perhaps related to the fact that it is expected to be zero in the continuum. The 


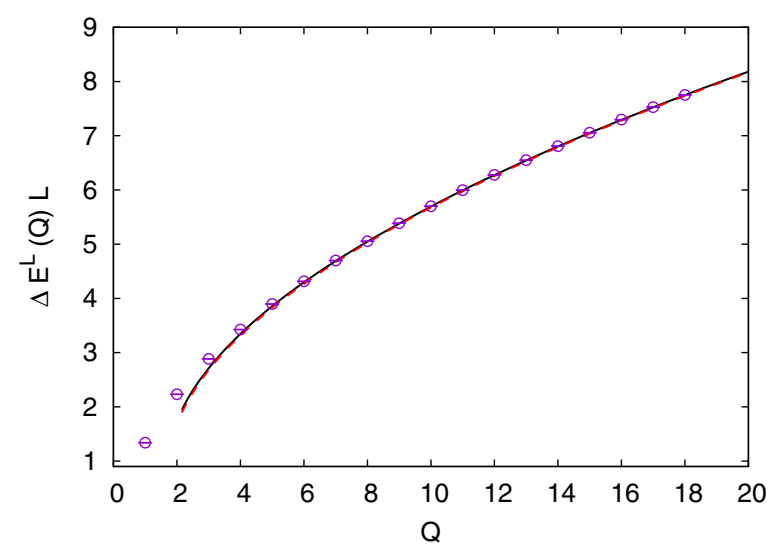

FIG. 4. The plot of $\Delta E^{L}(Q) L$ for $L / a=10$ given in Table III as a function of $Q$ along with a plot of the fitted function (solid line) with the fit parameters $c_{3 / 2}^{L}=1.235$ and $c_{1 / 2}^{L}=0.12$ and $c_{-1 / 2}^{L}=1.9$. The function with $c_{1 / 2}^{L}=0$ (dashed line) almost coincides with the solid line.

value of $c_{-1 / 2}$ is consistent with being nonzero, although it was recently argued that the classical contribution to the energy at this order is expected to vanish in the continuum limit [18]. Contributions from quantum corrections were not computed and could in principle be nonzero. Finally, as discussed in the Supplemental Material [36], all of our results are consistent with those obtained recently [12].

We wish to thank Uwe-Jens Wiese for helpful conversations. D. O. would like to thank Antonio Amariti, Simeon Hellerman, Orestis Loukas, and Susanne Reffert for enlightening discussions and comments. D. B. would like to thank Martin Hasenbusch, Ferenc Niedermayer, Rainer Sommer, and Ulli Wolff for useful discussions. The material presented here is based upon work supported by the U.S. Department of Energy, Office of Science, Nuclear Physics program under Award No. DE-FG02-05ER41368.

*debasish.banerjee@desy.de

†sch@phy.duke.edu

¥dorlando@itp.unibe.ch

[1] J. Cardy, in Exact Methods in Low-Dimensional Statistical Physics and Quantum Computing, Proceedings of the Les Houches Summer School, Session LXXXIX (Oxford University Press, Oxford, 2010).

[2] A. Pelissetto and E. Vicari, Phys. Rep. 368, 549 (2002).

[3] J. M. Maldacena, Int. J. Theor. Phys. 38, 1113 (1999); Adv. Theor. Math. Phys. 2, 231 (1998).

[4] A. B. Zamolodchikov, Pis'ma Zh. Eksp. Teor. Fiz. 43, 565 (1986) [JETP Lett. 43, 730 (1986)].

[5] J. Polchinski, Nucl. Phys. B303, 226 (1988).

[6] Y. Nakayama, Phys. Rep. 569, 1 (2015).

[7] S. Rychkov, EPFL Lectures on Conformal Field Theory in $D>=3$ Dimensions, SpringerBriefs in Physics (Springer, Cham, Switzerland, 2016).
[8] R. Rattazzi, V. S. Rychkov, E. Tonni, and A. Vichi, J. High Energy Phys. 12 (2008) 031.

[9] M. Campostrini, M. Hasenbusch, A. Pelissetto, P. Rossi, and E. Vicari, Phys. Rev. B 63, 214503 (2001).

[10] M. Campostrini, M. Hasenbusch, A. Pelissetto, P. Rossi, and E. Vicari, Phys. Rev. B 65, 144520 (2002).

[11] M. Schuler, S. Whitsitt, L.-P. Henry, S. Sachdev, and A. M. Läuchli, Phys. Rev. Lett. 117, 210401 (2016).

[12] S. Whitsitt, M. Schuler, L.-P. Henry, A. M. Läuchli, and S. Sachdev, Phys. Rev. B 96, 035142 (2017).

[13] J. L. Cardy, J. Phys. A 17, L385 (1984).

[14] J. L. Cardy, J. Phys. A 18, L757 (1985).

[15] S. Hellerman, D. Orlando, S. Reffert, and M. Watanabe, J. High Energy Phys. 12 (2015) 071.

[16] L. Alvarez-Gaume, O. Loukas, D. Orlando, and S. Reffert, J. High Energy Phys. 04 (2017) 059.

[17] A. Monin, D. Pirtskhalava, R. Rattazzi, and F. K. Seibold, J. High Energy Phys. 06 (2017) 011.

[18] O. Loukas, Fortschr. Phys. 65, 1700028 (2017).

[19] S. Hellerman, N. Kobayashi, S. Maeda, and M. Watanabe, arXiv: 1705.05825 .

[20] S. Hellerman, S. Maeda, and M. Watanabe, J. High Energy Phys. 10 (2017) 089.

[21] O. Loukas, D. Orlando, and S. Reffert, J. High Energy Phys. 10 (2017) 085.

[22] A. Monin, Phys. Rev. D 94, 085013 (2016).

[23] D. Jafferis, B. Mukhametzhanov, and A. Zhiboedov, arXiv: 1710.11161.

[24] D. T. Son and M. Wingate, Ann. Phys. (Amsterdam) 321, 197 (2006).

[25] M. Bordag, G. L. Klimchitskaya, U. Mohideen, and V. M. Mostepanenko, Int. Ser. Monogr. Phys. 145, 1 (2009).

[26] M. Hasenbusch and T. Török, J. Phys. A 32, 6361 (1999).

[27] M. Campostrini, M. Hasenbusch, A. Pelissetto, and E. Vicari, Phys. Rev. B 74, 144506 (2006).

[28] Y. J. Deng, H. W. J. Blote, and M. P. Nightingale, Phys. Rev. E 72, 016128 (2005).

[29] U. Wolff, Phys. Rev. Lett. 62, 361 (1989).

[30] D. Banerjee and S. Chandrasekharan, Phys. Rev. D 81, 125007 (2010).

[31] P. Calabrese, A. Pelissetto, and E. Vicari, Phys. Rev. B 67, 054505 (2003).

[32] M. De Prato, A. Pelissetto, and E. Vicari, Phys. Rev. B 68, 092403 (2003).

[33] J. M. Carmona, A. Pelissetto, and E. Vicari, Phys. Rev. B 61, 15136 (2000).

[34] M. Hasenbusch and E. Vicari, Phys. Rev. B 84, 125136 (2011).

[35] F. Kos, D. Poland, D. Simmons-Duffin, and A. Vichi, J. High Energy Phys. 11 (2015) 106.

[36] See Supplemental Material at http://link.aps.org/ supplemental/10.1103/PhysRevLett.120.061603 for contains technical details about the Monte Carlo algorithm used for the main results of the paper, the systematics of the different analysis methods, relevant finite size scaling, as well as comparison with existing results in the literature.

[37] D. Dantchev and M. Krech, Phys. Rev. E 69, 046119 (2004).

[38] M. Hasenbusch, J. Stat. Mech. (2008) P12006.

[39] M. Hasenbusch, J. Stat. Mech. (2009) P10006. 